\title{
CIF/IFC Continuing Forestry Education Program
}

Continuing education is a professional responsibility, and with rapid changes occurring in forest management and technology, particularly in relation to the environment and society there is pressure to keep up to date. The Canadian Institute of Forestry/Institut forestier du Canada recognizes that continuing forestry education is a shared responsibility of professional and technical associations, educational institutions, governments and employers and has developed a Continuing Forestry Education Program for CIF, RPF and nonmembers.

To obtain a "Continuing Forestry Education Certificate" applicants must have completed 150 hours of "approved" CFE activities within a three-year period. As of June 1995 there was a threeyear retroactive phase-in period. In Alberta, Ontario and New Brunswick the program is administrated jointly with the Registered Professional Foresters Association. In these provinces, there may be slight differences in requirements.

Activities are described in three broad categories recognizing different $\mathrm{CFE}$ activities. Credit weight varies with the type of activity, and the "contact hour" is based on one hour of instruction or equivalent. Credit may be claimed for course work.

The three categories are:

Structure learning - minimum 60 contact hours, but one can take all 150 hours in this category. Includes workshops, seminars, conference.

Self-improvement - 90 contact hours maximum, includes attendance at CIF/IFC or business meetings, reading or viewing/listening to related technical forestry material such as The Forestry Chronicle or videos.

Professional contributions - 60 contact hours maximum, includes giving presentations, writing articles (only when beyond the normal work duties), holding an elected or appointed office in CIF, RPF or allied professional organizations. You could be on your way to obtaining a CFE certificate.

Contact your local CIF/IFC office, or the RPF office in Alberta, Ontario and New Brunswick for more information.

\section{Forestry Continuing Education Certificates}

Congratulations to the following individuals for completing the requirements for the Continuing Education Certificate. Amirault, Peter, New Brunswick

Archibald, J. Harry, Alberta

Avery, Bruce, Alberta

Balkwill, Brian, Alberta

Barnhardt, Leonard, Alberta

Blackwood, Rick, Alberta

Brinker, Curtis, Alberta

Carnell, Brian, Alberta

Christie, Morley, Alberta

Ciesielski, Albert, Alberta

Clark, Kim, Alberta

Clowater, Wayne, New Brunswick

Cormier, Gerry, New Brunswick

Cosco, John, Alberta

Couturier, Gilles, New Brunswick

Davies, Brian, Alberta

Edey, Colin, Alberta

Ehrentraut, Garry, Alberta

Ferster, Rick, Alberta

Foley, Lou, Alberta

Folkmann, Paul, Alberta

Fullarton, Michelle, New Brunswick

Glover, Kenneth, Alberta

Golec, Patricia, Alberta
Grundberg, Byron, Alberta

Hache, Normand, New Brunswick

Hallett, Ron, New Brunswick

Hammond, James, Alberta

Harvey, Mark, Alberta

Henderson, John, New Brunswick

Hoffman, Thomas, Alberta

Hovan, Alwyn, Alberta

Johnson, Faye, Ontario

Kavalinas, Stan, Alberta

Kaytor, Larry, Alberta

Kent, Dave, Alberta

Kesseler, Robert, Alberta

Kuhnke, Dieter, Alberta

Lakusta, Thomas, Alberta

Lewis, Roxanne, Alberta

Luchkow, Steve, Alberta

MacLeod, David, Alberta

Macnutt, Peter, New Brunswick

Mann, Kim, New Brunswick

Mercer, Val, New Brunswick

Mohamed, El-Bayoumi, New Brunswick

Mullin, Tim, New Brunswick

Northey, Barry, Alberta

Nyrose, Craig, Alberta

O'Byrne, Marty, Alberta

Oberle, Frank, Alberta
Oldham, Hap, New Brunswick

Panas, Darrell, Alberta

Paver, Darby, Alberta

Presslee, David, Alberta

Quinn, John, Alberta

Quinn, Christine Kreibom, Alberta

Robertson, Sharon, Alberta

Rothwell, Richard, Alberta

Sieusahaim, Permanand, Alberta

Simpson, Dale, New Brunswick

Smith, Ron, New Brunswick

Smith, Darrell, Alberta

Somerville, Fred, New Brunswick

Splinter, Michael, Alberta

Stordock, Larry, Alberta

Swift, Ed, New Brunswick

Telfer, Edmund, Alberta

Tomchick, Robert, Ontario

Topolinski, Kevin, New Brunswick

Tosh, Kathy, New Brunswick

Udell, Robert, Alberta

Ullrich, Harry, Alberta

Wilson, Dale, New Brunswick

Wilson, Beverly, Alberta

Woodard, Paul, Alberta

The following individuals completed the requirements for two CFE certificates:

Bamsey, Colin, Alberta

Bickell, David, Alberta

Duane, Calvin, Alberta

Gelinas, Donald, Alberta

Greenhorn, Lorne, Alberta

Henteleff, Marke, Alberta

Johnson, Wayne, Alberta

Lehn, Gordon, Alberta

Newstead, Robert, Alberta

Patterson, David, Alberta

Renaud, Diane, Alberta

Rooks, Daniel, Alberta

Scatcherd, Doug, Alberta

Walker, H. Douglas, Alberta

Wearmouth, Patrick Currey, Alberta

Wilkinson, Daniel, Alberta

The following individuals completed the requirements for three CFE certificates:

Blake, Peter, Alberta

Friesen, Jim, Alberta

Gagne, Kevin, Alberta

Guidera, Patrick, Alberta

Hall, Ronald, Alberta

Macklin, Lois, Alberta

Murphy, Peter, Alberta

Roxanne Comeau, CIF/IFC Executive

Director - 2 April 1996 